\title{
Characteristics of the Filtering Bleb and the Agreement between Glaucoma Specialist and Anterior Segment-Optical Coherence Tomography Assessment
}

\author{
Fabio Zantut ${ }^{\mathrm{a}}$ Carolina Pelegrini Barbosa Gracitellia, ${ }^{\mathrm{a}}$ Paulo H. Souza ${ }^{\mathrm{a}}$ \\ Sergio H. Teixeira ${ }^{a}$ Augusto Paranhos Jr. ${ }^{a}$ \\ aDepartment of Ophthalmology and Visual Science, Federal University of São Paulo, São Paulo, Brazil; b Glaucoma \\ Service, Ver Mais Oftalmologia, Vinhedo, São Paulo, Brazil
}

\section{Keywords}

Glaucoma · Filtering bleb · Trabeculectomy · Anterior segment-optical coherence tomography

\begin{abstract}
Introduction: This study aimed to evaluate the characteristics of the filtering bleb in failed late trabeculectomy and the agreement between glaucoma specialist assessment and anterior segment-optical coherence tomography (AS-OCT) system assessment in terms of the presence of subconjunctival fluid in the filtering bleb. Methods: Patients with a diagnosis of glaucoma who were scheduled to undergo trabeculectomy in at least one eye and with uncontrolled intraocular pressure (IOP) were enrolled. All participants underwent a complete ophthalmological examination. The presence of fluid under the filtering bleb was first evaluated by a glaucoma specialist using biomicroscopy evaluation and then using the OCT 1000 AS-OCT Version 3.0.1.8 (Carl Zeiss Meditec, Dublin, (A, USA) system. A Kappa statistical test was used to evaluate the agreement between AS-OCT and the examiner. The correlation between conjunctiva and tenon thickness and clinical parameters was also assessed. Results: Forty eyes of 40 patients were evaluated in this study. The
\end{abstract}

ages ranged from 21 to 86 years, with a mean of $66.55 \pm$ 12.33. The average IOP was $21.20 \pm 4.44 \mathrm{~mm} \mathrm{Hg}$ (range 14-38 $\mathrm{mm} \mathrm{Hg}$ ) in the entire group. The mean thickness of the conjunctiva and tenon was $302.03 \pm 406.76 \mu \mathrm{m}$ (range 251$1,616 \mu \mathrm{m})$. There was a significant negative correlation between the mean thickness of the conjunctiva and tenon and IOP ( $p=0.045$; confidence interval $=-0.558,0.024)$. Additionally, there was a significant negative correlation between the mean thickness of the conjunctiva and tenon, and the number of medications used at baseline ( $p=0.043$; confidence interval $=-0.538,0.051)$. There was significant negative correlation between the horizontal measurement of the bleb and the use of glaucoma medications ( $p=0.017$; confidence interval $=-0.560,0.004)$. A total of 26 patients were determined to have fluid by the examiner (glaucoma expert), and the presence of fluid in AS was confirmed in 19 patients by AS-OCT. Of the 14 patients who were determined to have an absence of fluid, this was confirmed by AS-OCT in 7 patients (Kappa $=0.231$; agreement of $65.00 \%$ ). Conclusion: There is fair agreement between glaucoma specialist assessment and AS-OCT assessment in terms of the presence of fluid in trabeculectomy. Our findings highlight the importance of AS-OCT in some patients before deciding upon a new intervention.

(c) 2020 S. Karger AG, Base karger@karger.com

www.karger.com/ore

(C) 2020 S. Karger AG, Base

Karger"
Carolina Pelegrini Barbosa Gracitelli

Federal University of São Paulo, Rua Botucatu

821, Vila Clementino

São Paulo 04023-062 (Brazil)

carolepm@gmail.com 


\section{Introduction}

Trabeculectomy remains the gold standard surgical procedure for patients with glaucoma and clinically uncontrolled intraocular pressure (IOP). It is a highly effective technique, but it is associated with high rates of early and late failure [1]. The main cause of failure appears to be subconjunctival fibrosis along the episcleral bleb [2]. Failed trabeculectomy leaves the ophthalmologist with limited options for IOP control. An increase in the number of IOP lowering medications leads to increased morbidity and costs and may not be effective in controlling the disease. A second trabeculectomy may be technically complicated, and success rates are lower [3].

In patients who do not achieve the target IOP in the post-operative period after trabeculectomy, careful evaluation using biomicroscopy of the filtering bleb is crucial to decide the next course of treatment [4]. The presence or absence of fluid under the conjunctiva is not always clearly determined from the slit lamp, so this creates inefficiencies; however, knowing this, as well as the analysis of other characteristics such as encapsulated, hyperaemic, or flat blebs, can facilitate a better decision [5]. Biomicroscopy can lead the examiner to determine that the aqueous humour flow is close to a specific suture, and these findings may help when deciding which sutures should be removed [6]. In patients undergoing needling or surgical revisions, the IOP seems to further decrease in the presence of aqueous humour in the filtering bleb [7].

There are other tests that can help in the analysis of the filtering bleb, such as ultrasound biomicroscopy (UBM) [8]. The internal characteristics of the filtering bleb, such as fluid reflectivity and the bleb walls, can be characterised using UBM. However, UBM is limited by the need for contact and its low resolution of $25 \mu \mathrm{m}$. An alternative, anterior segment-optical coherence tomography (AS-OCT), offers better bleb imaging because it provides a non-contact approach for bleb assessment, it requires no coupling medium or supine positioning, it is contactless, and it is a high-resolution tomographic imaging technique of the cross-section of anterior segment structures leading a better analysis of the bleb. Previous studies have reported that AS-OCT performed by trained staff may show different characteristics of the filtering bleb, such as suprascleral fluid measurements, bleb walls, scleral flap, aqueous humour pathway, and iridectomy $[9,10]$.

In this context, this is the first study evaluating the agreement between the glaucoma specialist and the ASOCT in the assessment of the presence of fluid in the filtering bleb in failed trabeculectomy. In addition, as a sec-

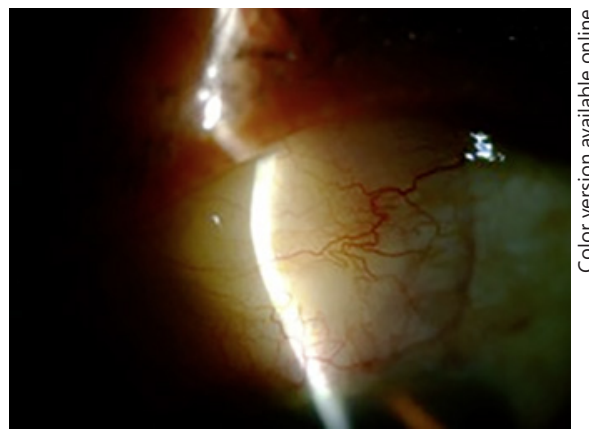

Fig. 1. Biomicroscopy image of the filtering bleb with fluid analysed by a glaucoma specialist.

ondary objective of the study, we evaluated the measurement of the late filtering bleb using AS-OCT and assessed the correlation with the IOP, and the number of ocular hypotensive medications used.

\section{Materials and Methods}

This cross-sectional observational study adhered to the tenets of the Declaration of Helsinki and was approved by the Institutional Review Board of the Federal University of São Paulo (CEP/ UNIFESP no. 834.138). Additionally, written informed consent was obtained from all participants.

\section{Patients}

We enrolled consecutive patients diagnosed with primary open-angle glaucoma who were scheduled for primary and repeat trabeculectomy (following failure) from the glaucoma division of the Federal University of São Paulo (São Paulo, Brazil). All participants underwent a complete ophthalmological examination that included review of medical history, best-corrected visual acuity, slit-lamp biomicroscopy, IOP measurement with Goldmann applanation tonometry (Haag-Streit, Koeniz, Switzerland), refraction, gonioscopy, and dilated fundoscopy examination. The inclusion criteria were (1) primary trabeculectomy $>1$ year prior and (2) lack of achievement of the target IOP even with topical medications. Patients who had other causes of trabeculectomy failure (that were not caused by changes in the bleb), such as iris entrapment or osteon problems, were excluded. Patients were also excluded if they (1) showed an orbital, corneal, or any ocular disease that could affect measured values of the IOP, (2) had a history of intraocular surgery, besides uneventful phacoemulsification, (3) had a history of ocular trauma, or (4) did not cooperate during ophthalmological examination.

The same experienced physician (i.e., a glaucoma specialist for $>10$ years) evaluated the presence or absence of fluid under the failed filtering bleb using a slit-lamp (anterior biomicroscopy) evaluation, and his evaluation was compared to the AS-OCT assessment. Figure 1 shows an example of the biomicroscopy of the filtering bleb of one of our patients. Regarding the biomicroscopic technique, the examiner first used wide and diffuse light with low 


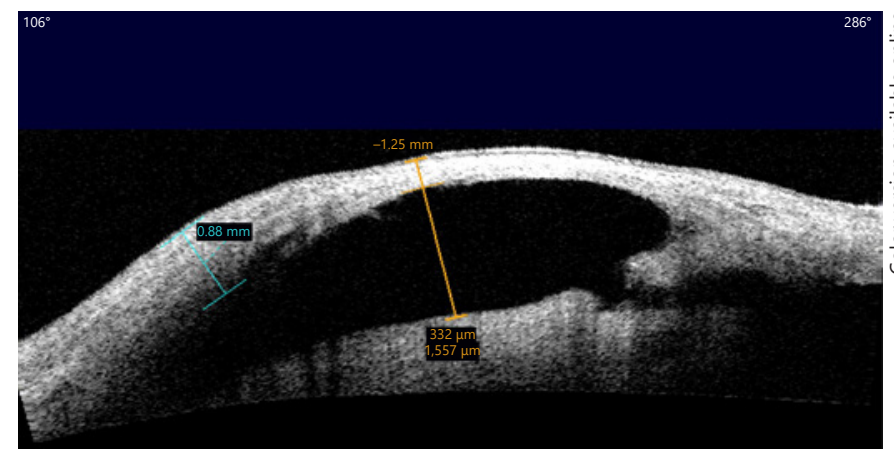

Fig. 2. AS-OCT image of the filtering bleb with fluid and its measures. AS-OCT, anterior segment optical coherence tomograph.

magnification to identify the scleral flap site and then a narrow optical cut of 1-2 millimetres angled with high magnification to observe the presence or absence of subconjunctival fluid.

AS-OCT 1000 (Carl Zeiss Meditec; Dublin, CA, USA) was used to confirm the absence or presence of fluid in the filtering bleb. This method has already been described to allow the visualization of the subtenonian or subconjunctival fluid with clarity as well as determination of its absence $[9,11]$. AS-OCT was performed by a trained ophthalmic technician who was blinded to the classification made by the glaucoma specialist (absence or presence of fluid in the filtering bleb). Measures of the conjunctiva and tenon thickness were obtained by longitudinal section on the site where the bleb was higher using a flap tool. The size of the vertical bleb (radial perpendicular to limbus) was determined using the transversal section in the greater area from the upper episcleral margin until the inner margin of conjunctiva. The horizontal bleb (tangential to limbus) measurement was made using the longitudinal section in the biggest area from the inner marginal conjunctiva in both lateral sides crossing the whole bleb with callipers. The measures were performed manually by an expert ophthalmic technician who was blinded to clinical data. Figure 2 illustrates the AS-OCT image of the bleb.

\section{Statistical Analysis}

A descriptive analysis was used to present demographic and clinical data. Descriptive statistics included mean and standard deviation values for normally distributed variables, while those not normally distributed were presented as median and interquartile range. Skewness/Kurtosis tests and histograms were used to check normality. Spearman's coefficient was used to analyse the correlation between the conjunctiva and tenon thickness with IOP as well as the conjunctiva and tenon thickness with the number of hypotensive medications used by patients. Multivariate analyses were performed to evaluate the effect of medication on correlation between conjunctiva and tenon thickness with IOP.

A Kappa statistical test was used to evaluate the agreement between the AS-OCT and the examiner. Kappa is a measure of interobserver agreement and measures the degree of agreement beyond that expected by chance alone. This measure of agreement has a maximum value of 1 , which represents total agreement, and values close to and $<0$ indicate no agreement or that agreement was exactly as expected by chance. All statistical analyses were per-

OCT and Slit Lamp: Evaluation of a Filtering Bleb
Table 1. Demographic findings in the entire group

\begin{tabular}{lc}
\hline Baseline characteristics & $\begin{array}{l}\text { Entire group, } \\
N=40 \text { eyes, } 40 \text { patients }\end{array}$ \\
\hline Age \pm SD, years & $66.55 \pm 12.33$ \\
Gender, $n(\%)$ & $19(48)$ \\
$\quad$ Female & $21(52)$ \\
$\quad$ Male & $10(25)$ \\
Race, $n(\%)$ & $30(75)$ \\
$\quad$ Black & $17(42)$ \\
$\quad$ Other & $21.20 \pm 4.44$ \\
Phakic (yes) & $3.15 \pm 0.74$ \\
IOP \pm SD, mm Hg & \\
Medications (abs values), $n$ &
\end{tabular}

IOP, intraocular pressure.

formed with commercially available software (Stata, version 13; StataCorp LP, College Station, TX, USA). The a level (type I error) was set at 0.05 .

\section{Results}

Forty eyes of 40 patients were enrolled in this study. The ages ranged from 21 to 86 years, with a mean of 66.55 \pm 12.33 years. The average IOP was $21.20 \pm 4.44 \mathrm{~mm} \mathrm{Hg}$ (range 14-38 $\mathrm{mm} \mathrm{Hg}$ ) in the entire group. All patients were using at least 2 classes of hypotensive eye drop, and 3 patients were using oral carbonic anhydrase inhibitor (acetazolamide). Table 1 summarizes the demographic findings in the entire group.

Regarding patient characteristics evaluated by ASOCT, the mean thickness of the conjunctiva and tenon was $302.03 \pm 406.76 \mu \mathrm{m}$ (ranges $251-1,616 \mu \mathrm{m}$ ), the mean diameter of the vertical bleb was $206.19 \pm 339.39 \mu \mathrm{m}$ (ranges $0-1,384 \mu \mathrm{m}$ ), and the mean diameter of the horizontal bleb was $1,570 \pm 2,260 \mu \mathrm{m}$ (ranges $0-9,770 \mu \mathrm{m}$ ). There was a significant negative correlation between the mean thickness of the conjunctiva and tenon and IOP $(p=0.045$; confidence interval $=-0.558,0.024)$. Additionally, there was a significant negative correlation between the mean thickness of conjunctiva and tenon, and number of medications used by patients ( $p=0.043$; confidence interval $=-0.538,0.051)$. However, when an adjustment was made for the number of medications, the mean thickness of the conjunctiva and tenon did not correlate with IOP $(p=0.118$, confidence interval $=-51.828$, 6.065 ; bivariate regression). Regarding the size of the bleb, we found a significant negative correlation between 
Table 2. Description of Kappa distribution in the entire sample

\begin{tabular}{lll}
\hline $\begin{array}{l}\text { AS-OCT } \\
\text { Glaucoma expert }\end{array}$ & $\begin{array}{l}\text { Presence of } \\
\text { fluid, yes (\%) }\end{array}$ & $\begin{array}{l}\text { Presence of } \\
\text { fluid, no (\%) }\end{array}$ \\
\hline $\begin{array}{l}\text { Presence of fluid, yes (\%) } \\
\text { Presence of fluid, no (\%) }\end{array}$ & $\begin{array}{c}19(48) \\
7(18)\end{array}$ & $7(18)$ \\
\hline
\end{tabular}

Kappa $p$ value $=0.231$ agreement of $65.00 \%$.

the horizontal measurement of the bleb and the use of glaucoma medications $(p=0.017$; confidence interval $=$ $-0.560,0.004)$.

A total of 26 patients were determined to have fluid by the examiner (glaucoma expert), and of these patients, the presence of fluid was confirmed in 19 by AS-OCT. On the other hand, from glaucoma expert evaluation, 14 patients were determined to have an absence of fluid, and 7 of these patients had confirmation of absence by OCT (Kap$\mathrm{pa}=0.231$; agreement of $65.00 \%)$. Between the 14 patients with disagreement, 8 had cyst intraconjunctival and 6 have not $(p>0.05)$. Table 2 describes the Kappa distribution in the sample.

\section{Discussion}

This study describes the morphology of the bleb as measured by AS-OCT and its correlation with IOP and number of medications in patients with failed late trabeculectomy. The present study found a fair agreement between the glaucoma specialist and AS-OCT in terms of the assessment of the presence of fluid after trabeculectomy. In addition, there was a significant correlation between the mean thickness of the conjunctiva and tenon and IOP and the number of medications. However, when an adjustment was made for the number of medications, the mean thickness of the conjunctiva and tenon did not correlate with IOP. We also found a correlation between the size of the horizontal bleb and the number of medications; however, the correlation became insignificant after adjusting for the number of medications.

Although trabeculectomy is effective in most cases [1], it is necessary for some patients to perform an additional procedure to reach the target IOP. Lysis of suture, massage, needling, or surgical revision techniques may be necessary after the surgery [12-14], and the use of intraoperative antimetabolites such as 5-fluorouracil and mitomycin Chas been shown to bring better results to this technique [15]. However, according to previous research, the success rates for trabeculectomy usually vary from 60 to $90 \%$ [12-14]. In terms of post-operative management, the presence of fluid and its location may influence the clinical decision when the target IOP is not reached. For example, Rotchford and King [7], in a prospective study evaluating 81 consecutive patients who underwent bleb needling, showed that the bleb morphology can be used to predict success in recent, highly vascularized or microcystic trabeculectomy blebs. A bleb which is still in the remodelling phase (early after trabeculectomy intervention) or that retains some degree of function (microcystic changes) can predict a good prognosis. Lin et al. [16] showed that after 3 years, most patients required a new procedure, and the findings obtained from AS-OCT could provide valuable information to the physician in terms of the new intervention. Different approaches have been suggested to better analyse the bleb appearance. For example, Kapetansky and Kapetansky [17] evaluated the appearance of blebs in 30 eyes before mitomycin $\mathrm{C}$ using UBM. They showed that success was higher in cases where the bleb was present preoperatively, which supports the importance of knowing about the presence of fluid in the filtering bleb. The AS-OCT, which has been shown to be able to determine the presence of fluid in the post-operative period [18], together with a close clinical examination, may play an important role in cases where the presence of fluid may influence the outcome. Waibel et al. [11] showed that AS-OCT was superior to the examiner in determining the encapsulation characteristics of the filtering bleb within 4 weeks post-operatively, corroborating the difference in the evaluation of the filtering bleb between the examiner and the AS-OCT. However, Leung et al. [9] found a good correlation between examination with the slit-lamp and AS-OCT in classifying the characteristic patterns of the filtering bleb, such as encapsulated, cystic, and flat. Oh et al. [19] showed correlation between slit-lamp examination and AS-OCT and concluded that a higher bleb with an increased aqueous reservoir is associated with optimal IOP results, and these findings corroborate with ours in terms of the bleb features and IOP.

Our findings regarding bleb morphology agree with Leung et al. [9]. In this study, diffuse filtering blebs were found by subconjunctival fluid collection. Suprascleral fluid space and the route under the scleral flap were found in 4 of the 7 patients. Cystic blebs were composed of a large hyporeflective space with multiloculated fluid collections covered by a thin layer of conjunctiva. Encapsulated blebs had a thick bleb wall with high reflectivity and 
an enclosed fluid-filled space. Flattened blebs demonstrated high scleral reflectivity and no bleb elevation. A similar approach was used by Narita et al. [20], and they showed similar results. We found the least need for medication in patients with larger horizontal blebs, but in patients with thinner conjunctiva (encapsulated), we observed a lower IOP control, which was also seen in patients without liquid under the conjunctiva.

In our study, the agreement between the AS-OCT and the examiner was fair. It is likely that the elevation of the surgical site caused by subconjunctival fibrosis can mimic the presence of fluid and this may have been the main cause of the result found between AS-OCT and examiner. However, in our sample for all patients with disagreement between AS-OCT and glaucoma specialist, almost half had cyst intraconjunctival and the other half did not.

The most relevant finding in our study was that there was a fair agreement between the glaucoma specialist and AS-OCT regarding presence of fluid in trabeculectomy. As far as we know, this is the first study comparing glaucoma expert assessment and that of an image tool. The fact that there was a fair agreement represents an enormous clinical finding. However, as it is possible that ophthalmologists will sometimes need machines to better evaluate the morphology of filtering blebs because even a highly trained glaucoma expert may fail to detect fluid in failed trabeculectomies, meaning that in some patients, we should be using AS OCT instead of attempting to guess if fluid is present.

Usually, the morphology of the filtering bleb is correlated with its function. Therefore, careful post-operative examination to enable early decision-making and intervention may improve the final results of surgery. Our study also revealed that experienced clinicians can accurately detect the presence of fluid; however, the auxiliary AS-OCT may be unusual in some cases. However, we emphasise that the presence of subconjunctival fluid is not necessarily synonymous with good functioning since we can have an encapsulated bleb with subconjunctival fluid and high IOP [21].

It is important to address some limitations of this study. First, only one examiner performed the AS-OCT imaging. Therefore, we did not perform a test-retest analysis of the measurement results. However, this examiner was masked to the clinical evaluation results and this person had several years of experience. Second, other characteristics of the bleb were not evaluated in this study that could affect the agreement results. Third, in our sample, patients had trabeculectomy that did not reach the target IOP, surgeries occurred $>1$ year ago, and hypotensive eye drops were used. These characteristics may have influenced the biomicroscopic analysis and may not represent patients with other characteristics.

In conclusion, the present study reports the characteristics of bleb measurements in failed trabeculectomy and the agreement between glaucoma specialists and ASOCT. We found a fair agreement between glaucoma specialist assessment and AS-OCT assessment in terms of the presence of fluid in trabeculectomy. Our findings suggest the importance of AS-OCT in some patients before deciding upon a new intervention.

\section{Statement of Ethics}

This study adhered to the tenets of the Declaration of Helsinki and was approved by the Institutional Review Board of the Federal University of São Paulo (CEP/UNIFESP no. 834.138). Additionally, written informed consent was obtained from all participants.

\section{Conflict of Interest Statement}

The authors have no conflicts of interest to declare.

\section{Funding Sources}

The authors did not receive any funding.

\section{Author Contributions}

Conceived and designed the experiments: F.Z., C.P.B.G., P.H.S., S.H.T., and A.P.J. Performed the experiments: F.Z., C.P.B.G., P.H.S., S.H.T., and A.P.J. Analysed the data: F.Z., C.P.B.G., P.H.S., and A.P.J. Contributed reagents/materials/analysis tools: F.Z., C.P.B.G., P.H.S., and A.P.J. Contributed to the writing of the manuscript: F.Z., C.P.B.G., P.H.S., and A.P.J.

References

1 Feldman RM, Tabet RR. Needle revision of filtering blebs. J Glaucoma. 2008;17(7):594600.

2 Skuta GL, Parrish RK. Wound healing in glaucoma filtering surgery. Surv Ophthalmol. 1987;32(3):149-70.

3 Shirato S, Kitazawa Y, Mishima S. A critical analysis of the trabeculectomy results by a prospective follow-up design. Jpn J Ophthalmol. 1982;26(4):468-80.

4 Purtskhvanidze K, Saeger M, Treumer F, Roider J, Nölle B. Long-term results of glaucoma drainage device surgery. BMC Ophthalmol. 2019;19(1):14. 
5 Maestrini HA, Cronemberger S, Matoso HD, Reis JR, Mérula RV, Filho AD, et al. Late needling of flat filtering blebs with adjunctive mitomycin C: efficacy and safety for the corneal endothelium. Ophthalmology. 2011;118(4): 755-62.

6 Keicher AS, Stoffelns BM. Argon laser suture lysis following glaucoma filtering surgery: a short introduction to the procedure. Med Laser Appl. 2010;25(4):209-13.

7 Rotchford AP, King AJ. Needling revision of trabeculectomies bleb morphology and longterm survival. Ophthalmology. 2008;115(7): 1148-e4.

8 Pavlin CJ, Harasiewicz K, Foster FS. Ultrasound biomicroscopy of anterior segment structures in normal and glaucomatous eyes. Am J Ophthalmol. 1992;113(4):381-9.

9 Leung CK, Yick DW, Kwong YY, Li FC, Leung DY, Mohamed S, et al. Analysis of bleb morphology after trabeculectomy with Visante anterior segment optical coherence tomography. Br J Ophthalmol. 2007;91(3): $340-4$.

10 Golez E 3rd, Latina M. The use of anterior segment imaging after trabeculectomy. Semin Ophthalmol. 2012;27(5-6):155-9.
11 Waibel S, Spoerl E, Furashova O, Pillunat LE, Pillunat KR. Bleb morphology after mitomycin-c augmented trabeculectomy: comparison between clinical evaluation and anterior segment optical coherence tomography. J Glaucoma. 2019;28(5):447-51.

12 D'Ermo F, Bonomi L, Doro D. A critical analysis of the long-term results of trabeculectomy. Am J Ophthalmol. 1979;88(5):829-35.

13 Layden W. Longterm results of trabeculectomy in the Japanese: an analysis by life table method. Surv Ophthalmol. 2004.

14 Ramakrishna S, Nelivigi S, Sadananda AM, Ganesh S. Study of efficacy and timing of laser suture lysis in reducing intraocular pressure after trabeculectomy with mitomycin-C. Oman J Ophthalmol. 2016;9(3):144-9.

15 Chen CW. Enhanced intraocular pressure controlling effectiveness of trabeculectomy by local application of mitomycin-C. Trans Asia Pac Acad Ophthalmol. 1983;9:172-7.

16 Lins S, Byles D, Smith M. Long-term outcome of mitomycin $\mathrm{C}$-augmented needle revision of trabeculectomy blebs for late trabeculectomy failure. Eye. 2018;32(12):1893-9.
17 Kapetensky FM, Kapetensky SD. Antimetabolite use in revising failing filtering blebs. Semin Ophthalmol. 1999;14(3):144-51.

18 Teus MA, Paz Moreno-Arrones J, Castaño B, Castejon MA, Bolivar G. Optical coherence tomography analysis of filtering blebs after long-term, functioning trabeculectomy and $\mathrm{XEN}^{\star}$ stent implant. Graefes Arch Clin Exp Ophthalmol. 2019;257(5):1005-11.

19 Oh LJ, Wong E, Lam J, Clement CI. Comparison of bleb morphology between trabeculectomy and deep sclerectomy using a clinical grading scale and anterior segment optical coherence tomography. Clin Exp Ophthalmol. 2017;45(7):701-7.

20 Narita A, Morizane Y, Miyake T, Seguchi J, Baba T, Shiraga F. Characteristics of successful filtering blebs at 1 year after trabeculectomy using swept-source three-dimensional anterior segment optical coherence tomography. Jpn J Ophthalmol. 2017;61(3):253-9.

21 Yarangumeli A, Koz OG, Kural G. Encapsulated blebs following primary standard trabeculectomy: course and treatment. J Glaucoma. 2004;13(3):251-5. 